\title{
6 Logische und realphilosophische Kategorien
}

In Bezug auf das Programm der Logik sind die logischen von den realphilosophischen Kategorien zu unterscheiden, wie sie jenseits der Logik in der „Realphilosophie“ erörtert werden. Denn jene sind als einzelne weitaus weniger „autonom“ als die realphilosophischen Kategorien, da sie vollständig nur als „Momente der absoluten, unhintergehbaren Struktur“36 der absoluten Idee vorkommen. Sie werden dahingehend als „rein“ gedacht, als sie von allen besonderen, empirisch-gegenständlichen Gehalten frei sind und in gut aristotelischer Tradition das Seiende überhaupt, insofern es Seiendes ist, begrifflich entfalten ${ }^{37}$. Gerade die letztgenannte Hinsicht ist von besonderem Interesse: meint sie doch den Umstand, dass die logischen Kategorien allem Seienden, „um es denken zu können, vorausgesetzt werden. "38 Damit aber umreißen und bestimmen sie als Ganzes, d.h. im notwendigen Zusammenhang ihres Entwicklungsprozesses, wie sie letztendlich in der "absoluten Idee“ als Prozess aufgehoben werden ${ }^{39}$, den „Raum“40 dessen, was überhaupt möglich und wirklich sein kann. Seiendes, gleich welcher weiteren Bestimmtheit, kann nur in diesem ontologischen Feld und ge-

36 Hösle: Hegels System, S. 72. Dass die logischen Kategorien, wie Hösle schreibt, „rein begrifflicher Art“ sind, also „kein Pendant in der Vorstellung“ (Hösle: Hegels System, S. 72) haben, stimmt zwar, trifft aber nicht ausschließlich auf diese zu.

37 Vgl. Iber: Metaphysik absoluter Relationalität, S. 9.

38 Hösle: Hegels System, S. 72.

39 Dass es in Hegels Logik nicht bloß um die Veränderung von Begriffen innerhalb von Prozessen geht, sondern auch die Prozesse des Denkens selbst als sich selbst aufhebende Prozesse gedacht werden müssen, d.h. die als Prozesse gedachten Gedankenbewegungen sich beim Denken des Denkens also auch selbst aufheben, zeigt Stephan Siemens: „Nichts - Negation - Anderes. Eine Kritik an Henrichs ,Formen der Negation in Hegels Logik““, in: Jahrbuch für Hegelforschung (2010), Bd. 12, hg.v. Helmut Schneider, S. 225-266, hier: S. 254.

40 „Raum“ ist hier nicht die realphilosophische Kategorie des physikalischen Raums, sondern Metapher für das ontologische wie epistemologische „Worin“ und „Wie“ des „Seins überhaupt“. Gemeint ist damit im Rahmen der Logik, die sich ja als eine Wissenschaft versteht, eine Art „Theorierahmen“ (vgl. Carnap: Empirismus, Semantik und Ontologie, S. 27), ein „Gesamtnetz“ [total network] (Quine: Two Dogmas, S. 41), ein „logischer Raum“ als „die Gesamtheit dessen, was der Fall sein und gedacht werden könne“ (Koch: Die Evolution des logischen Raumes, S. 1). Dass dieser logische Raum bei Hegel keineswegs vom Standpunkt vorhegelianischer „Standard-Metaphysiken“ als „eine fertige, statische, ewige Gegebenheit“ (Koch: Die Evolution des logischen Raumes, S. 4), sondern in seiner Prozessualität zu verstehen ist, begründet den „evolutionären“ Charakter seiner Logik: „Hegel ist [...] der Entdecker der Evolution und Prozessualität des logischen Raumes, und seine neue oder Nicht-Standard-Metaphysik ist die zugehörige Evolutionstheorie, eine, wenn man so will, evolutionäre Logik.“ (Koch: Die Evolution des logischen Raumes, S. 4)

Ә OpenAccess. (c) 2021 Claudia Wirsing, publiziert von De Gruyter. (cc) BY unter einer Creative Commons Namensnennung 4.0 International Lizenz. 
mäß dessen fundamentalen Regelsystemen des Bestimmtseins und ZueinanderVerhaltens als denkmöglich und wirklich angesprochen werden: Hegel spricht demzufolge von der Metaphysik der Logik als von einem „diamantene[n] Netz, in das wir allen Stoff bringen und dadurch erst verständlich machen“ (TWA 9, S. 20, $\S 246$ [Zusatz]). Dieser ontologischen Intention der logischen Kategorien entspricht ihre Selbstreferenz, die von ihrer Selbstbezüglichkeit unterschieden werden muss. ${ }^{41}$ Selbstreferenziell sind sie nämlich, weil sie aufgrund ihrer strengen Allgemeinheit „selbst unter das fallen, was sie bedeuten“42, d. h. zugleich Exemplar und Gattung, Realisierung und Begriff sind: Die Kategorie des Seins ist selbst ein Sein, die Kategorie des Etwas selbst ein Etwas etc. Demgegenüber meint Selbstbezüglichkeit eine Eigenschaft, die nicht alle Kategorien innehaben, oder zumindest nicht in gleich expliziter Weise bzw. als Funktionsweise ihres begrifflichen Gehalts: Auf dem Stand der Wesenslogik bspw. ist „Unmittelbarkeit“ eine negativ-selbstbezügliche Kategorie, d. h. sie bedeutet eine reflexive Struktur, wohingegen „Sein“ oder „Nichts“ in der Seinslogik das zumindest ihrer Selbstbeschreibung nach nicht tun. Selbstreferenz ist eine Seinsbeziehung im Sinne einer Selbstverwirklichung (energeia/actualitas), Selbstbezüglichkeit eine Wissensbzw. Bedeutungsbeziehung: Selbstreferenziell ist etwas darin, was es aussagt (Seinsbeziehung); selbstbezüglich sagt etwas darüber aus, was es ist (Wissensbeziehung). Die allgemeine Selbstreferenz der Kategorien besteht gerade darin, auch sich unter sich zu subsumieren. Eben darin zeigt sich der grundsätzliche transgressive Charakter logischer Kategorien, der in ihrer absoluten Vorgängigkeit auch gegen sich selbst besteht. Die logischen Kategorien sind vorgängig gegenüber jedem besonderen Gebrauch oder jeder besonderen Reflexion über sie, weil sie in ihrem Gebrauch wie in ihrer Bestreitung, ob auf sich oder auf Gegenstände überhaupt bezogen, stets schon in Anwendung sind. Damit geben sie ebenfalls einen Hinweis darauf, wie jeder kategoriale Begriff von „Realsein überhaupt“, will er Sinnvolles aussagen, die sich als Objekt selbst verortete Vorgängigkeit begrifflicher Formen und damit die Aufhebung des abstrakten Unterschieds eines Reichs der Begriffe und eines Reichs der Gegenstände anerkennen muss, ohne zugleich in eine abstrakte Identität beider zurückzufallen.

Dementsprechend koinzidiert die Logik mit den Ansprüchen der Ontologie dergestalt, dass „nur in seinem Begriffe [...] etwas Wirklichkeit“ (GW 21, S. 34) hat und die rein logische Aufklärung über den inneren und äußeren Zusammenhang von Denkbestimmungen nicht sinnvoll in einem Verhältnis der Andersheit zu den

41 Vgl. dazu Hösle: Hegels System, S. 72-74.

42 Hösle: Hegels System, S. 73: „Wenn sie wirklich allgemeine Kategorien sind, so kann es nichts geben, was nicht unter sie fällt; also müssen sie auch von sich selbst ausgesagt werden.“ 
Begriffen des Wirklichseins zu denken ist. ${ }^{43}$ So zeigt sich auch im ontologischen Anspruch der Logik die Voraussetzung der denknotwendigen Vorgängigkeit begrifflicher Strukturen innerhalb des Realen, ohne dass doch beide so zusammenfallen, dass ein selbstständiges „Reales überhaupt“ jenseits der begrifflichen Operationen des Denkens geleugnet werden würde. Im Verlauf der Logik kommt alles darauf an, den Unterschied objektiv-begrifflicher Formationen und subjektiv-begrifflicher Operationen von Verstand und Vernunft sowohl in ihrem Unterschied als auch in ihrer Verbundenheit verständlich zu machen, um zu begründen, warum sich auf kategoriale Weise „Realität überhaupt“ nicht anders denken lässt als im inneren Zusammenhang dieses Unterschieds.

Geht man aufgrund dieser Voraussetzung von einem vollständigen logischen Begriff der Wirklichkeit aus und nimmt diesen ins Visier, so ist dieser nur mit der Logik im Ganzen zu identifizieren. Nur der Gesamtzusammenhang aller Kategorien des Seienden bis zu seinem Resultat, der diesen Gang in sich aufhebenden „Idee“, mithin der ganze Gang der Logik vom Sein zur Idee inklusive deren Rückgang in den Anfang, beschreibt hegelianisch vollständig das, was in logischer Perspektive über die begriffliche Struktur der Wirklichkeit - ihre notwendigen und wahrhaften begrifflichen Muster - gesagt werden muss. ${ }^{44}$ Unsere Frage aber ist in doppelter Hinsicht anders ausgerichtet: Erstens fragt die Arbeit nach den im engeren Sinn kategorialen Strukturen und Bedingungen von „Realität überhaupt“. Damit erkundet sie in der Tradition kategorialer Reflexion die begriffslogischen Minimalbedingungen dessen, was „Realsein überhaupt“ heißt, noch jenseits einer vollständigen logischen Erkundung aller hinreichenden Bedingungen von Realität in ihrer absoluten begrifflichen Entfaltung und gemäß eines besonderen Systems wie demjenigen Hegels. Dass alle Begriffe der Logik für einen vollständigen logischen Begriff der Wirklichkeit notwendig sind, heißt eben nicht, dass sie gleich notwendig für einen darin zu findenden und aus ihm zu gewinnenden Minimalbegriff von „Realsein überhaupt“ sein müssen. Gerade hier ist Hegels organologisches Metaphernsystem für seine Systematik unbedingt ernst zu nehmen: Das Ganze und Vollentwickelte der Idee findet sich in jedem Systemteil auch im Besonderen und Noch-nicht-Vollendeten, auf eigensinnige Weise perspektiviert, als Ganzes wieder; es kommt sich immer wieder selbst in den logischen Gestalten seines graduellen Ausstands innerhalb des Systems zuvor und fügt sich so mit sich in den Stadien seiner abnehmenden Defizienz zusammen. Deshalb kann der erste Teil der Wesenslogik so verstanden werden, dass hier das Ganze der

43 Vgl. Hösle: Hegels System, S. $69 \mathrm{f}$.

44 Vgl. Hösle (Hegels System, S. 69), der andeutet, wie der vollständige Begriff jedes Einzeldings nur durch den Zusammenhang aller Kategorien $\mathrm{zu}$ bestimmen ist. 
Idee als Inbegriff des vollständigen logischen Wirklichseins bei Hegel die Perspektivierung auf seine - zwar gemessen am Ganzen defizienten, trotzdem kategorial sinnvollen - Minimalbedingungen wahrhaften Realseins erstmals ermöglicht. Zweitens ist das Interesse der Arbeit grundsätzlich ein systematisches; damit aber muss sie dezidiert Abstand davon nehmen, einen Theorierahmen vollständig einzunehmen, welcher in der historischen Abfolge untersucht wird. Das Ziel ist es, mithilfe der Rekonstruktion eines historischen Problemzusammenhangs, aber grundsätzlich jenseits einzelner diskutierter Theorieangebote und ihrer weitreichenden Bedingungen und Konsequenzen ein begriffliches Angebot dafür zu erarbeiten, „Realsein überhaupt“ kategorial-minimal verständlich zu machen. Philosophie bewährt sich dort, wo sie es möglich macht, kategoriale Probleme aufzuzeigen und lösbar zu machen, ohne den gesamten Theorierahmen ihrer Diskussion im Ergebnis vorauszusetzen und mitzuführen. Dementsprechend ist es das Ziel, in der Reflexion bestimmter Stadien in Hegels Logik die kategoriale Frage der sinnvollen Minimalbestimmung von „Realsein überhaupt“, verstanden als grundbegriffliche Matrix des An-sich-Bestehenden, in Bezug auf den kantischfichteschen Problemstand einer Antwort zuzuführen. Die vorliegende Arbeit will zeigen, dass Hegels Logik dafür in der Tat eine überzeugende Lösungsmöglichkeit bereithält, die die inneren Widersprüche und Unbestimmtheiten bei Kant und Fichte aufnimmt und durchreflektiert. Sie vertritt jedoch nicht die Ansicht, dies müsse notwendig, gemäß dem hegelschen Programm, einzig in der Integration der Logik als ganzer in diesen gesuchten kategorialen Begriff geschehen, oder gar unter Annahme der gesamten Philosophie Hegels. Entsprechend setzt die hier zu entwickelnde Argumentation auch nicht die Gültigkeit des Prinzips voraus, jede Denkbestimmung im logischen Voraus der „absoluten Idee“ sei in verschiedenen Graden begrifflich defizient und deshalb erst das Ganze der Logik im Begriff der „Idee“ philosophisch hinreichend entfaltet und damit brauchbar. Zwar wird natürlich das Evolutionsprinzip der Denkbestimmungen, d.h. ihr defizitärer Charakter in Bezug auf das, was sie jeweils begrifflich ausdrücken wollen, und in Bezug darauf, in welcher Weise bzw. gemäß welcher formaler Prinzipien sie dies tun, ernst genommen. Die Logik der Gesamtsequenz der Kategorien ist jedoch dabei in zweifacher Weise einzuschränken: zum einen auf die Kategorien, welche im engeren Sinn (und zwar historisch präfiguriert als auch systematisch ausgezeichnet) Kandidaten für die begriffliche Beschreibung des „Realseins überhaupt“ darstellen. Zum anderen kann die logische Defizienz dieser Kategorien bis zu dem Punkt verfolgt werden, an welchem diese in Bezug auf unsere Fragestellung hinreichend nicht-defizient genug sind, um das besondere Problem eines Minimalbegriffs von „Realsein überhaupt“ adäquat erfassen zu können. Dies aber, so soll gezeigt werden, ist im ersten Kapitel der Wesenslogik eben der Fall. Hegels kategoriale Minimalbestimmung von „Realität überhaupt“ fällt also nach 
meiner Lesart weder mit einer einzelnen Kategorie der Logik ${ }^{45}$, noch mit ihrem Gesamtzusammenhang, und weder mit ihrem Anfang noch mit ihrem Ende zusammen: Vielmehr wird sie lokal an einer genau bestimmten Stelle innerhalb der Logik, aber in einem Darstellungszusammenhang mehrerer kategorialer Gedankenbestimmungen (Wesen - Schein - Reflexion) als metakategoriales Potenzial entwickelt. Die Logik nicht nur von ihrer teleologischen Vollständigkeit her als unauftrennbare Einheit zu lesen, sondern auch von anderen Kriterien her als offenere Synthese, ist z. B. auch durch den Umstand gerechtfertigt, dass ihre generelle und für die gesamte Logik gültige Methode der „Einheit von Darstellung und Kritik“46, „,in der die Denkbestimmungen sich ,selbst untersuchen“ und ,ihren Mangel aufzeigen““647 sollen, durch eine zweite, deutlich desintegrativere Ordnung ergänzt wird: „Allerdings haben die einzelnen Teile der hegelschen Logik - die Logik des Seins, des Wesens und des Begriffs - zur Metaphysik eine unterschiedliche Stellung. Nicht die Logik schlechthin, bloß die ,objektive‘, welche die Seinslehre und die Lehre vom Wesen umfaßt, sollen an die Stelle der vormaligen Metaphysik treten. “48 Demnach verläuft eine Trennlinie zwischen objektiver und subjektiver Logik anhand der Funktion der Kritik und ihres Gegenstandes ${ }^{49}$ : Die objektive Logik ist mit der Kritik und sukzessiven Ersetzung der alten Metaphysik beschäftigt, die subjektive Logik vornehmlich mit der Erfüllung der neuen in ihrer logischen Vollständigkeit. Im Verhältnis wiederum von Seinslogik zu Wesenslogik innerhalb der objektiven Logik nimmt die Wesenslogik, emblematisch deutlich in ihrem ersten Satz sichtbar, den Status ein, die „Wahrheit“ der alten Metaphysik zu enthalten, d.h. deren Fragen in den Zustand ihrer Wahrheit zu überführen, nachdem die Seinslogik vor allem die ungenügenden Antworten der alten Metaphysik kenntlich gemacht hat. Diese Fragen der alten Metaphysik jedoch, so Hegel, welche durch die neue kritische Metaphysik der objektiven Logik nicht als Fragen aufgelöst, sondern im Gegensatz zur alten Metaphysik nur auf wahrhafte Weise - im Rahmen der Wahrheitsmöglichkeiten dieser Fragen - beantwortet

45 Dass eine solche Zuweisung zu unlösbaren Problemen führt, hat das Kapitel zu Kants Kritik der reinen Vernunft (Kap. 1 im Hauptteil I) gezeigt.

46 Theunissen: Sein und Schein, S. 14. Vgl. zum methodischen Konzept des „Zuschauens“, das dabei zum Tragen kommt, auch Claudia Wirsing: „Das reine Zusehen. Absolute Bildung in Hegels Wissenschaft der Logik“, in: Bildung der Moderne, hg.v. Michael Dreyer, Michael N. Forster, KaiUwe Hoffmann, Klaus Vieweg, Tübingen 2013, S. 181-196.

47 Theunissen: Sein und Schein, S. 15.

48 Theunissen: Sein und Schein, S. 24.

49 Wie weitreichend diese Trennlinie ist, wie fest oder scheinhaft, ist sicherlich Gegenstand verschiedener Meinungen, aber dass diese Trennlinie existiert, wohl nicht. Damit aber kann sie in jedem Fall Begründungsfunktion für Unternehmungen haben, die sich auf einzelne potenzielle Aspekte von Hegels Logik konzentrieren. 
werden sollen, sind solche, die „die Natur des En s überhaupt erforschen“ (GW 21, S. 48): also die Frage nach dem Wesen des Seienden als Seiendes, des „Realen überhaupt“. Es gilt zu entscheiden, ob man den Übergang der Wesens- zur Begriffslogik so versteht, als würden sich nun nicht mehr nur die Antworten der alten Metaphysik (wie sie in der Seinslogik abgebildet sind) als obsolet herausstellen, sondern als würden sich auch bereits die Fragen der alten Metaphysik nach dem Ens auflösen - oder ob man die neue Wissenschaft des Begriffs in der Begriffslogik (auch) als eine komplementäre Wissenschaft versteht, welche eben einzig die Fragehorizonte, die durch die Metaphysik der objektiven Logik (auch in ihrer wahrhaften wesenslogischen Form) nicht beantwortet oder gar nicht gestellt werden können, in einer neuen logischen Wissenschaft ergründet. Diese zweite Ansicht, welche hier vertreten wird, versteht die Defizite der Wesenslogik, wie sie vor allem in deren zweiten Teil erscheinen und den Übergang in die Begriffslogik vorbereiten, nicht als auflösende bzw. die Seins- und Wesenslogik und ihre Fragen gänzlich verabschiedende, sondern einfach als Hinführung zu anderen Frage- und Denkgebieten des Logischen, die sich dann seins- oder wesenslogisch nicht mehr angemessen beschreiben lassen, sich zwar aus den Problembeständen der Seinsund Wesenslogik ergeben, aber zugleich nicht einfach deren begriffliche Muster in jeder Hinsicht obsolet machen. Die Wesenslogik wird durch die Begriffslogik nicht obsolet gemacht, sondern sie enthält die weiterhin gültigen Antworten auf die alten Fragen der Metaphysik nach dem Wesen des Ens, wie sie in der Seinslogik erscheinen. Die Begriffslogik dagegen formuliert nicht mehr die Antworten der alten Metaphysik um, sondern sie beginnt zumindest mit anderen Fragen, nämlich denen nach den kategorialen Gehalten der Vernunfttätigkeiten (Begriff, Urteil, Schluss). Deshalb ist der genetische Zusammenhang von Seins- und Wesenslogik auf dieser Ebene enger als der der Seins- und Wesenslogik zur Begriffslogik; ein Unterschied, der gelegentlich in der Forschung thematisiert wird. ${ }^{50}$

Die Größe und Komplexität der Logik liegt m. E. gerade darin, nicht einsinnig und einsträngig bestimmte metaphysische Fragen und Antworten in ihrem Fortgang diffamierend gänzlich zu verabschieden, indem sie im Prozess der sie tragenden Kategorien aufgehoben werden, sondern auch jeweils die Geltungsbedingungen und Geltungsmöglichkeiten verschiedener metaphysischer Ordnungen und Beschreibungssysteme, wie sie vom Sein bis zum Begriff auftreten, aufzuzeigen. Dass diese teleologisch geordnet sind und sich auseinander als

50 Vgl. Christian Iber: „Hegels Konzeption des Begriffs“, in: G.W.F. Hegel. Wissenschaft der Logik, hg.v. Anton Friedrich Koch und Friedrike Schick, Klassiker Auslegen, Bd. 27, Berlin 2002, S. 181201, hier: S. 181. 
Architektur höherstufiger Prozessualität bis hin zur „absoluten Idee“ ergeben, bedeutet nicht, dass sie einander deshalb im Fortgang nur zum Verschwinden bringen. Anders gesagt: Die Logik darf nicht so verstanden werden (die differenzierende Dimension von „Aufhebung“ sträflich missachtend), als würden vorhergehende Kategorien, kategoriale Themenfelder und Stufen einfach nur überschrieben werden und damit quasi überholt. Denn das würde die absurde Konsequenz erzeugen, dass die Logik ein dickes Buch mit dünnem Resultat wäre: Wirklich ,wahr‘ und ,funktionstüchtig' wäre demnach nur das letzte Kapitel als Ergebnis, in dem alle vorhergehenden Kapitel verschwunden sind und das selbst nicht mehr derart defizitär ist, dass es von einem weiteren Schritt ,verbessert werden müsste. Die anhaltende Aktualität der Logik besteht m. E. viel eher in dem Umstand, in den verschiedenen Teilen, Kapiteln und Abschnitten vom reinen Sein zur absoluten Idee neben der teleologischen, formal eher sukzessiv-zeitlichen Struktur der Überschreibung unangemessenerer durch angemessenere Kategorien zugleich eine räumliche Ordnung von logischen Problembeschreibungsprozeduren zu finden, die nebeneinander bestehen können und trotz ihrer je verschiedenen philosophischen Ausarbeitung und Problemanfälligkeit für bestimmte Fragestellungen weiterhin Gültigkeit besitzen. Das leuchtet schon von der philosophischen Praxis her ein, die Hegel auch stets im Auge hatte: Bestimmte Fragen benötigen Begrifflichkeiten mit einer höheren, genaueren und komplexeren Auflösung; andere wiederum, ohne dass sie dadurch bereits obsolet wären, kommen mit einfacheren, in mancherlei Hinsicht vielleicht problematischeren Begrifflichkeiten aus, die aber möglicherweise auf diese Fragen viel passender zugeschnitten und viel funktionaler ausgerichtet als höhere, komplexere begriffliche Instrumente sind. Das bedeutet deshalb nicht, dass die Antworten in diesem Rahmen weniger komplex sind, aber ihre Explikation benötigt die Detailbezogenheit, die einzelnen Kategorien und den damit verbundenen Problemkomplexen zukommt. Genau in diesem Sinn soll hier das „Erste Kapitel“ der Wesenslogik in Bezug auf die Frage nach der Beschreibung minimaler kategorialer „Realität überhaupt“ als innerhalb der Logik angemessenster Schlüssel verstanden werden, auch wenn dieses Kapitel nicht ,die“ Wahrheit der Logik überhaupt bereitstellt bzw. seine Begrifflichkeiten selbst nicht in anderen Hinsichten kritikwürdig sind. Für den begrenzten Rahmen unserer Fragestellung also, die nicht den Zusammenhang der „absoluten Idee“ im Ganzen im Blick hat und diesen zu interpretieren unternimmt, sondern lediglich die Frage nach den metaphysischen kategorialen Minimalbedingungen von „Realität überhaupt“ stellt (und damit die Frage danach als grundsätzlich sinnvoll erachtet), ist es gerade die Wesenslogik, die es zu untersuchen gilt. Dabei kommt deren Anfangskapitel wiederum eine besondere Bedeutung zu, weil es erstens die reinen logischen Vollzugsformen des Wesens analysiert - und noch nicht deren Produkte (Reflexionsbestimmungen) - 
also gewissermaßen die Grammatik des Wesens enthält, und zweitens noch nicht in den Resultaten des Wesens bereits die Bedingungen entfaltet, welche seine Defizienz aufzeigen und demnach in die Logik des Begriffs hinüberführen.

Im Folgenden soll deshalb also der Vorschlag unterbreitet werden, das schon von mehreren Interpreten in verschiedenen Zusammenhängen als besonders gewichtig fokussierte Kapitel „Der Schein“ zu Anfang der Wesenslogik, welches die Reflexionsformen des Wesens entfaltet und $\mathrm{zu}$ dessen Reflexionsbestimmungen übergeht, in eben dieser Hinsicht als eigentliches Zentrum einer Reflexion auf die kategoriale Minimalform von „Realität überhaupt“ zu lesen und die Kategorienentwicklung im logischen Davor (Seinslogik) und Danach (spätere Wesenslogik, Begriffslogik) als bezogen auf die Durchdringung eben dieses Kerngedankens zu verstehen. Immerhin hebt Hegel die Wesenslogik in der Enzyklopädie dadurch hervor, als „(der schwerste) Theil der Logik“ (GW 20, S. 145, § 114) gelten zu dürfen: eine Kennzeichnung, die nicht nur eine der Darstellungsform, sondern auch eine der Sache ist, und die anhand der Forschung anzeigt, welche sich überlagernden Bedeutungsschichten an ihr freizulegen sind. Gerade das „Erste Kapitel“ des „Ersten Abschnitts“ der Wesenslogik also („Der Schein“), welches in der Forschung so differente Bewertungen erfahren hat ${ }^{51}$ und das Hegel noch dazu in der Kurzfassung der Wesenslogik, in der Enzyklopädie, beinahe gänzlich auslässt bzw. auf wenige Sätze zusammenschmilzt, wird in minimaler realitätskategorialer Hinsicht als Schaltstelle und Zentrum der gesamten Logik betrachtet: also nicht, wie bspw. bei Dieter Henrich, als esoterisches Methodenkapitel der Logik, sondern vielmehr als spekulative Entfaltung der kategorialen Grundstruktur jedes sinnvollen philosophischen Zugriffs auf den philosophischen Begriff der Realität.

51 Vgl. Dieter Henrich: „Hegels Logik der Reflexion“, in: Ders.: Hegel im Kontext, Berlin 2010, S. 95-157, hier: S. 122f. John M. E. McTaggert (A commentary on Hegel’s logic (1910), Reprint, Bristol 1990, S. 99) hat wirkmächtig eben die scheinbare Isolation und funktionale Desintegration des „Schein“-Kapitels als argumentative Schwäche gelesen und demzufolge die straffere Struktur der Enzyklopädie für vorteilhafter erklärt: „The three categories of the triad of Show - Essential and Unessential, Show, and Reflection, find no place in the Encyclopedia, where the Doctrine of Essence starts with the category of Identity. In this the later work seems to me much superior to the earlier.“ Iber (Metaphysik absoluter Relationalität) wiederum liest das erste Kapitel ebenfalls als Zentrum der Logik, allerdings als Erörterung der ontologischen Metaphysik absoluter autonomer Relationalität. Zur problematischen Frage (gegen Henrich), ob es sich bei Hegels Erörterungen dieses Kapitels wirklich um Methodenüberlegungen handelt und ob überhaupt die dialektische Grundstruktur der Reflexion darin sinnvoll als „Methode“ bezeichnet werden kann, vgl. Michael Wolff: „Hegels Dialektik - eine Methode? Zu Hegels Ansichten von der Form einer philosophischen Wissenschaft“, in: Hegel. 200 Jahre Wissenschaft der Logik, hg.v. Anton Friedrich Koch, Friedrike Schick, Klaus Vieweg, Claudia Wirsing, Hamburg 2014, S. 71-86. 
Methodologisch könnte man zum Verständnis dieses Zugriffs analogisch-erläuternd (d.h. als illustrative Stütze) auf Walter Benjamins, im Übrigen in großer Sachnähe zu Hegels Logik entworfene ${ }^{52}$ Philosophie der „Idee“ in der „Erkenntniskritischen Vorrede“ verweisen. Benjamin bestimmt dort die temporale Logik der Idee im Rückgriff auf den Modus des „Ursprungs“ folgendermaßen:

Im Ursprung wird kein Werden des Entsprungenen, vielmehr dem Werden und Vergehen Entspringendes gemeint. Der Ursprung steht im Fluß des Werdens als Strudel und reißt in seine Rhythmik das Entstehungsmaterial hinein. Im nackten offenkundigen Bestand des Faktischen gibt das Ursprüngliche sich niemals zu erkennen, und einzig einer Doppeleinsicht steht seine Rhythmik offen. Sie will als Restauration, als Wiederherstellung einerseits, als eben darin Unvollendetes, Unabgeschlossenes andererseits erkannt sein. ${ }^{53}$

Die tragende Gestalt und das prinzipielle Zentrum des logisch Wesentlichen des minimalen kategorialen Begriffs von „Realität überhaupt“, so könnte man die Fragestellung mit Benjamin verständlich machen, stehen nicht einfach am logischen Anfang oder Ende des vollständigen begrifflichen Zusammenhangs der Idee. Vielmehr finden sie sich positional eingegliedert innerhalb der ,Evolution“ des Kategorialen (,im Fluß des Werdens“) und doch zugleich wie ein senkrechter Schnitt quer dazu in einer horizontalen Struktur (,dem Werden und Vergehen Entspringendes“). Als „Strudel“ etabliert diese Position eine zweite Ordnung neben der linear aufsteigenden Progression vom Sein zur Idee. Gerade der Charakter des „Unvollendete[n], Unabgeschlossene[n]“ der Reflexionsformen in Bezug auf die lineare Ordnung des gesamten logischen Zusammenhangs, in der sie eben nicht das letzte Wort darstellen, garantiert dabei ihre besondere bedeutungslogische Strahlkraft: weil sie so eine bestimmte Art der noch offenen Potenzialität ${ }^{54}$ beherbergen, die befreit ist von der Last, das Ganze der Logik an sich ausbilden zu

52 Vgl. dazu ausführlich die originelle und sehr fundierte Studie von Jan Urbich (Darstellung bei Walter Benjamin. Die ,Erkenntniskritische Vorrede` im Kontext Ästhetischer Darstellungstheorien der Moderne, Berlin/Bosten 2012, S. 147-157). Entgegen der immer noch gängigen Auseinandersetzung Benjmains mit der kantischen Philosophie, hat Jan Urbich als erster überhaupt das philosophisch Gemeinsame von Benjamins und Hegels Metaphysik systematisch und detailiert aufgeschlüsselt. Vgl. hierzu paradigmatisch auch seine Walter-Benjamin-Vorlesungen an der Universität Girona (Jan Urbich: Benjamin and Hegel. A Constellation in Metaphysics. Walter Benjamin-Lectures at the Càtedra Walter Benjamin, Girona 2016).

53 Walter Benjamin: „Ursprung des deutschen Trauerspiels“, in: Ders.: Abhandlungen. Gesammelte Schriften. Bd. I.1, Frankfurt am Main 1991, S. 203-430, hier: S. 226.

54 Hans Heinz Holz hat dementsprechend den benjaminschen Begriff des Ursprungs auch als Markierung des „Aktualisierbaren“ an bestimmten theoretischen Beständen verstanden (Hans Heinz Holz: „Prismatisches Denken“, in: Über Walter Benjamin, Frankfurt am Main 1968, S. 62111, hier: S. 65). 
müssen, und so als einzelnes Glied innerhalb der Logik zugleich in besonderer Weise die Erklärungskompetenz gleichwohl vollendeter minimaler Bedingungen $\mathrm{zu}$ beherbergen vermögen. Das so mithilfe von Hegels enormer logischer Problemlösungskompetenz erreichte Mindestset einer begrifflichen Norm des Realseins mag nicht für ein Gesamtprojekt wie das der Logik Hegels, wohl aber für unsere Fragestellung ausreichend entwickelt und begründet sein. Es soll nicht vollständig abgelöst werden von den hegelschen Bedingungen seiner Erarbeitung - wohl aber übertragbar sein auf begriffliche Programme anderer philosophischer Theorierahmen. Ein solches Vorgehen versucht also die Mitte zu wahren zwischen einer reflexionslosen und gewaltsamen Nutzung von historischen Theorieangeboten als Ideensteinbrüchen und der rein immanenten Rekonstruktion von diesen. Deshalb soll im Folgenden auch zuerst, entgegen der linearen zielgerichteten Ordnung der Logik, dieses realitätskategoriale Argumentationszentrum der Reflexionsformen ausführlich erläutert werden. 